\title{
Synthesis of silica-polymer core-shell hybrid materials with enhanced mechanical properties using a new bifunctional silane-based photoinitiator as coupling agent
}

\author{
Rita Zakhia Douaihy ${ }^{\mathrm{a}, 1}$, Igor Telegeiev ${ }^{\mathrm{a}, 1}$, Hussein Nasrallah ${ }^{\mathrm{a}}$, Oleg Lebedev ${ }^{\mathrm{b}}$, Philippe Bazin ${ }^{\mathrm{a}}$, \\ Alexandre Vimont ${ }^{\mathrm{a}}$, Jean-François Chailan ${ }^{\mathrm{c}}$, Armand Fahs ${ }^{\mathrm{c},}$ Mohamad EL-Roz ${ }^{\mathrm{a}, *}$ \\ a Normandie Université, ENSICAEN, UNICAEN, CNRS, Laboratoire Catalyse et Spectrochimie, 14050 Caen, \\ France \\ ${ }^{\mathrm{b}}$ Normandie Université, ENSICAEN, UNICAEN, CNRS, Laboratoire CRISMAT, 14050 Caen, France \\ ${ }^{c}$ Université de Toulon, Laboratoire MAPIEM, 83041 Toulon Cedex 9, France \\ ${ }^{1}$ Equal contribution \\ * Corresponding author
}

\begin{abstract}
Here, we report the use of new bifunctional silane-based type-1 photoinitiator (SPI-1) as a coupling agent for photopolymer filler and silica grafting. The SPI-1 is grafted on the surface of silica nanoparticles via interactions between the ethoxy group of the silane and the silanol groups of the silica surface. The grafted particles are then dispersed or embedded in/with acrylate polymer by a direct photopolymerization process. The materials were characterized using different techniques including UV-visible spectroscopy, FTIR, TGA, and TEM. Their mechanical properties and the surface morphology were also investigated using AFM and DMA analyses. A significant change and enhancement of the mechanical properties of the newly synthesized materials were observed with respect to that of the unmodified silica. The analysis of the morphology at the microscale level reveals interesting information on the origin of this enhancement and on the dispersion of the filler in the polymer matrix.
\end{abstract}

\section{Introduction}

Hybrid materials have received extensive interest and gained their effectiveness due to their astonishing characteristics and their dramatic improvements in the material properties. Particularly, hybrid inorganic-organic UV-curable materials have emerged to be good candidates in different application fields such as in microelectronics, photolithography for micro and macro 3D printing, plastics, adhesives and coatings[1-9]. The rapid, inexpensive and environmentally friendly synthesis of these materials is based on the treatment of a photosensitive substance, the so-called photoinitiator, using light radiation to promote the polymerization of the organic layer [10-12]. Moreover, during the free radical photopolymerization, photoinitiators absorb the appropriate wavelength, generate primary radical species by $\alpha$-cleavage (type I) or $\mathrm{H}$-abstraction (type II) depending on their type, and initiate therefore the polymerization [13-16]. However, various inert or 
active oxides (e.g. silica, $\mathrm{TiO}_{2}$, etc.) are used as filler (core) to attend the desired properties of the hybrid products. When none of the reactants is capable of absorbing the photochemically active light, the grafting of an active photoinitiator on the surface of the support becomes crucial [17]. In fact, modifying the surface of the core reactant has been widely used in thermal polymerization (e.g. rubber vulcanization) since it prevents the agglomeration of the core nanoparticles, inhibits clusters formation, assures thus a homogeneous dispersion and simultaneously controls the thickness, the density and the functionality of the formed polymer[18-20].

Silica nanoparticles are commonly used as fillers for hybrid inorganic-organic UV-curable materials[7-21-22]. Their importance is due to the fact that they are optically inert, low toxic, highly abundant, thermally and chemically stable and permeable to template molecules. Their surface is highly rich in silanol groups, so they are extremely hydrophilic and are not adequate for a homogeneous dispersion in the polymer matrix [23]. Grafting core active photoinitiators on the silica surface allows their good dispersion and assures their stability by increasing their hydrophobicity. Therefore, strong covalent chemical interactions are generated between them and the polymer matrix, respectively [24].

Herein, a newly synthesized silane-based bifunctional type I photoinitiator coupling agent (SPI-1) [25-26] is tested in the free-radical photopolymerization on the surface of silica nanoparticles. Particularly, SPI-1 is grafted on the surface of silica nanoparticles via interactions between the silane and the silanol groups on the silica surface. Then, highly active ketyl radicals are generated on the silica surface by direct fragmentation under UV irradiation. The efficiency of these grafted radicals is investigated in the photopolymerization of trimethylolpropane triacrylate (TMPTA). The hybrid materials are characterized using UV-visible and FTIR spectroscopies. The mechanical properties of the films are also exploited using AFM and DMA analyses. The reported results are compared with that obtained by using the parent polymer film. Finally, a photopolymerization in solution is tested to elucidate the mechanical properties and to examine the morphological aspects of the synthesized material.

\section{Materials and methods}

\subsection{Materials}

The chemicals, purchased from Aldrich, were used without any further purification: Isobutyrophenone (IBP, $97 \%$ ), Allyl bromide (99\%), Potassium t-butoxide (99.99\%), Decane ( $\geq 99 \%)$, Diethyl ether (99 $\%)$, Magnesium sulfate (99.99\%), Triethoxysilane (95\%) and Trimethylolpropanetriacrylate (TMPTA) (technical grade). Ultrasil $7000 \mathrm{GR}$ (Evonik-Degussa), with a surface area of $175 \mathrm{~m}^{2} / \mathrm{g}$ and a primary particle size of around $14 \mathrm{~nm}$, was used as silica filler.

\subsection{Methods}

\subsubsection{Synthesis of SPI-1}


The synthesis of 2,2-dimethyl-1-phenyl-5-(triethoxysilyl) pentane-1-one (SPI-1) is conducted in a twostep reaction (Scheme. 1) as described below.<smiles>CC(C)C(=O)c1ccccc1</smiles>
$90 \%$<smiles>C=CCC(C)(C)C(=O)c1ccccc1</smiles>

1

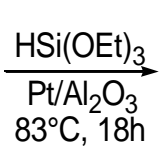

$40 \%$<smiles>CCOCCCC(C)(CCC[SnH])C(=O)c1ccccc1</smiles>

Scheme. 1. Schematic representation of the two-step reaction of the synthesis of SPI-1. NMR data of the precursor (1) and SPI-1 products are available in supplementary information (Fig. S1-S2).

To a solution of isobutyrophenone $(10 \mathrm{~g}, 67 \mathrm{mmol})$ and $t$-BuOK $(15,128 \mathrm{mmol})$ in $40 \mathrm{~mL}$ of $t-\mathrm{BuOH}$, $11 \mathrm{~mL}$ of allylbromide $(128 \mathrm{mmol})$ were added dropwise. The overall mixture was stirred for 18 hours at $83{ }^{\circ} \mathrm{C}$ and then poured into water and extracted with $\mathrm{Et}_{2} \mathrm{O}$. The combined organic extracts were dried over $\mathrm{Na}_{2} \mathrm{SO}_{4}$ and the solvent was removed at reduced pressure.

Later, a solution of triethoxysilane (685 uL, $3.7 \mathrm{mmol})$ ) and precursor $1(1 \mathrm{~g}, 5.3 \mathrm{mmol})$ was stirred with $1.5 \mathrm{~g}$ of $\mathrm{Pt} / \mathrm{Al}_{2} \mathrm{O}_{3} 5 \mathrm{wt} \%$ at $83^{\circ} \mathrm{C}$ without adding any solvent in a Schlenk tube. The reaction was conducted under $\mathrm{N}_{2}$ for 18 hours. Following that, the mixture was filtered and washed with absolute $\mathrm{EtOH}_{\mathrm{abs}}$ through activated charcoal. The final product was collected after concentration and dried by rotary evaporation.

\subsubsection{Grafting of SPI-1 on the surface of silica nanoparticles}

$1 \mathrm{~g}$ of silica nanoparticles was first dried at $150{ }^{\circ} \mathrm{C}$ and then dispersed in $20 \mathrm{~mL}$ of decane at $120{ }^{\circ} \mathrm{C}$ under vigorous stirring for 10 minutes. Later, $100 \mathrm{mg}$ of the previously synthesized SPI-1 was added. The overall mixture was stirred for 30 minutes. The powder was obtained by centrifugation and redispersed in water/ethanol solvent $(\mathrm{v} / \mathrm{v})$ to remove the excess of the ungrafted SPI-1 and the solvent. Before characterization, the white precipitate was dried at $50^{\circ} \mathrm{C}$ for 12 hours and stored in the dark.

\subsubsection{Photopolymerization Process}


The siloxane functions of the synthesized initiator can promote an efficient grafting of the molecule on the silica surface and the photosensitive group (benzoyl function) promotes the generation of free radicals by direct fragmentation under UV-irradiation (Scheme. 2). The UV light used to initiate the photopolymerization was generated by a polychromatic Xe-Hg lamp (LC8-01A spotlight, Hamamatsu, L10852, $200 \mathrm{~W}, \mathrm{I}_{0} \approx 100 \mathrm{~mW} . \mathrm{cm}^{-2}$ ) and was directed through a flexible fiberoptic. The prepared samples are take-free and the conversion degree is between 75 and $80 \%$ for all the samples.

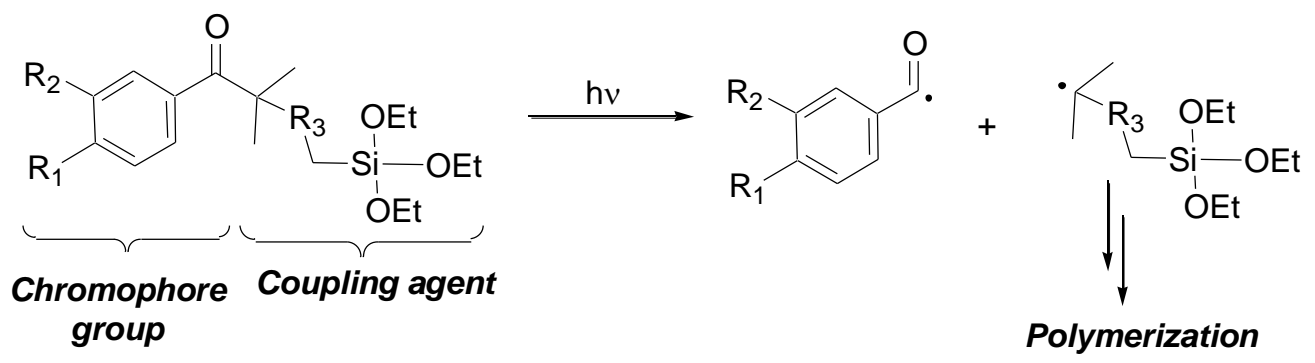

Scheme. 2. Schematic representation of the mechanism of the free radical generation under UV irradiation.

\subsubsection{Film preparation}

Prior to AFM and DMA analyses, three different films were prepared for comparison: 1) pure polymer film; 2) polymer film containing 10 wt.\% of ungrafted silica (silica@polymer) and prepared by adding 1 wt\% of external photoinitiator (IBP, Scheme. 3(B)); and 3) polymer film prepared by the dispersion of $10 \mathrm{wt} \%$ grafted silica (SPI-1/silica) without adding the external photoinitiator, designated as SPI1/silica@polymer film. The films were synthesized using a Teflon spacer of a width of $5 \mathrm{~mm}$ and a diameter of $50 \mathrm{~mm}$. Ungrafted and grafted silica nanoparticles were dispersed with the external photoinitiator and the monomer. Few drops of the mixture were spread on polypropylene films and subjected to UV irradiation.

(A)<smiles>CCO[SiH2]CCCC(C)(C)C(=O)c1ccccc1</smiles>

(B)<smiles>CC(C)C(=O)c1ccccc1</smiles>

Scheme. 3. Schematic representation of (A) SPI-1 and (B) Isobutyrphenone (IBP).

\subsubsection{Photopolymerization in solution}

$2 \mathrm{~mL}$ of acetonitrile $(\mathrm{AcN})$ mixed with few drops of the monomer trimethylolpropane triacrylate (TMPTA) was added to a transparent vial containing $100 \mathrm{mg}$ of SPI-1/Silica. Being one of the mostly used monomer, this trifunctional monomer can lead to a 3D polymer matrix as a model in this specific polymerization. The mixture was stirred and bubbled with argon for degassing. The photopolymerization was then carried out under polychromatic irradiation and soft stirring at room temperature for 120 seconds. After polymerization, the excess solvent was removed using 
centrifugation and the precipitate was washed several times with $\mathrm{AcN} / \mathrm{H}_{2} \mathrm{O}$ solution $(1 / 1, \mathrm{v} / \mathrm{v})$. The obtained precipitate was dried at $70{ }^{\circ} \mathrm{C}$ for more than 12 hours prior to characterization.

\section{Characterization techniques}

Diffuse reflectance UV-Vis (DR UV-Vis) measurements of the obtained powder (SPI-1/silica) were performed in the spectral range $200-900 \mathrm{~nm}$ with a Cary 4000 spectrophotometer (Varian), equipped with Praying Mantis Diffuse Reflection Accessory.

The thermogravimetric analysis was conducted on a SETSYS instrument (SETARAM) analyzer (25$800{ }^{\circ} \mathrm{C}$, a heating rate of $5{ }^{\circ} \mathrm{C} \cdot \mathrm{min}^{-1}$ under $40 \mathrm{~mL} \cdot \mathrm{min}^{-1}$ flow of air) to determine water and hydrocarbon contents in the grafted sample.

FTIR spectroscopy was performed on a $\sim 20 \mathrm{mg}$ of the powder pressed $\left(\sim 10^{7} \mathrm{~Pa}\right)$ into self-supported pellets $\left(2 \mathrm{~cm}^{2}\right.$ area) and placed in an IR cell equipped with $\mathrm{KBr}$ windows. IR spectra were recorded in the region between 400 and $5500 \mathrm{~cm}^{-1}$ at a resolution of $4 \mathrm{~cm}^{-1}$ and for $128 \mathrm{scans}$, using a Nicolet 6700 IR spectrometer equipped with a DTGS detector and an extended-KBr beam splitter.

The AFM measurements were carried out on a Multimode 8 microscope (Bruker) using the PeakForce Quantitative Nanomechanics Mapping mode (PF-QNM). The scans were recorded on three different regions on the films and the maps were collected with $256 \times 256$ pixels at a scan rate of $1 \mathrm{~Hz}$. The radius of the tip was estimated during the calibration step, was evaluated by the relative method on a polystyrene test sample, and was found to be equal to $\sim 50 \mathrm{~nm}$. Silicon probes were selected upon the recommendation of the AFM microscope producer, and the nominal spring constant of the cantilever was $40 \mathrm{~N} \cdot \mathrm{m}^{-1}$ (RTESPA-300). As for the DMA analyses, the experiments were performed on a TA Instrument DMA 2980 in a single cantilever mode. The dimensions of the sample were about $18 \mathrm{~mm} \times 10 \mathrm{~mm} \times 1 \mathrm{~mm}$ (length $\times$ width $\times$ thickness). A sinusoidal load, automatically adjusted to achieve a displacement of $10 \mu \mathrm{m}$ at the frequency of $1 \mathrm{~Hz}$, was applied to remain in the viscoelastic range. The storage modulus was recorded from the ambient temperature to $150{ }^{\circ} \mathrm{C}$ with a heating rate of $3^{\circ} \mathrm{C} \cdot \mathrm{min}^{-1}$.

The microstructure of the sample was examined using a transmission electron microscopy HAADFSTEM (High-angle annular dark field). TEM micrographs along with the elemental maps were recorded on JEM200F cold FEG double aberration corrected microscope equipped with CENTURIO large-angle EDX detector. Before that, the powder was dispersed in ethanol on a holey carbon film and coated with copper. Low-intensity beam conditions were applied during the measurements to minimize the degradation of the material under the electron beam 


\section{Results and discussion}

After grafting on the silica surface, the characteristic bands of the grafted SPI-1 are examined using diffuse reflectance DR UV-visible and transmission FTIR spectroscopies. The DR UV-visible spectrum for the SPI-1/Silica in Fig. 1(A) shows the presence of new bands centered at 315 and 276 $\mathrm{nm}$. These bands are attributed to the $n-\pi^{*}(315 \mathrm{~nm})$ and $\pi-\pi^{*}(230-300 \mathrm{~nm})$ electronic transitions of the benzoyl groups of the SPI-1, respectively. Additionally, the FTIR spectrum of the grafted silica sample in Fig. 1(B) displays new characteristic bands on the grafted silica pellets (summarized in Table S1 in the SI), thus confirming the presence of SPI-1 on the silica surface.

(A)

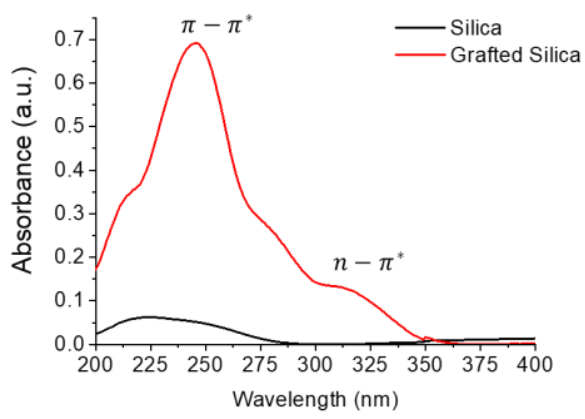

(B)

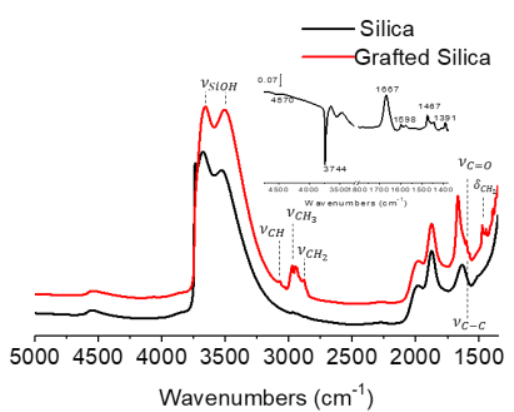

Fig. 1. (A) Diffuse reflectance UV-visible spectra and (B) FTIR spectra of pure silica nanoparticles and grafted silica with SPI-1. The inset in (B) corresponds to the subtraction result of the FTIR spectra of the grafted silica with SPI-1 from that of the parent silica. FTIR spectra are recorded at RT under vacuum after activation at $150^{\circ} \mathrm{C}$.

Thermogravimetric analysis (TGA) and in-situ FTIR with thermal programed desorption (TPD) are carried out to determine qualitatively and quantitatively the nature of the interactions between the SPI1 and the silica surface. The TGA curve of the grafted silica powder is compared to that of the pure silica powder (Fig. 2A). In both cases a first weight loss observed below $100{ }^{\circ} \mathrm{C}$ is attributed to the release of water and the desorption resulting from the condensation of the vicinal silanol groups (2 $\mathrm{SiOH} \rightarrow \mathrm{SiOSi}+\mathrm{H}_{2} \mathrm{O}$ ). As for the grafted silica sample, an additional weight loss is observed at relatively high temperature. It cannot be assigned to the condensation of supplementary silanol groups since the vicinal silanol content is similar in both samples (vicinal silanols are not active for grafting). It is then attributed to the thermal degradation of the grafted SPI-1 hydrocarbon. The derivative weight loss versus the temperature reveals three main peaks for SPI-1/Silica samples at $192{ }^{\circ} \mathrm{C}, 350^{\circ} \mathrm{C}$ and $465^{\circ} \mathrm{C}$ (Fig. 2B). The peak centered at $192{ }^{\circ} \mathrm{C}$ is assigned to the physisorbed SPI- 1 and represents less than $20 \%$ of the total SPI-1. The peaks at $350{ }^{\circ} \mathrm{C}$ and $465{ }^{\circ} \mathrm{C}$ are due to the degradation and the total oxidation of the strongly adsorbed SPI-1 species, respectively. Based on these results and considering the similar contents of water and silanol in the parent and in the grafted sample, the SPI-1 loading in this latter is estimated to $1.7 \pm 0.5 \mathrm{wt} \%$. 
(A)

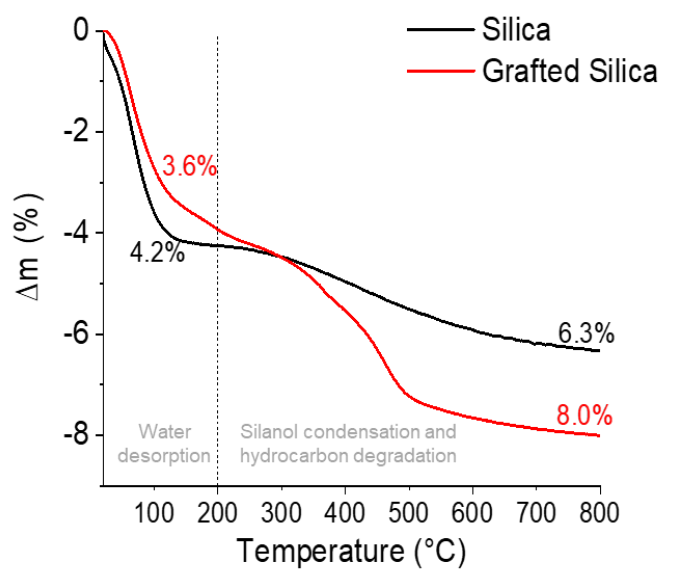

(B)

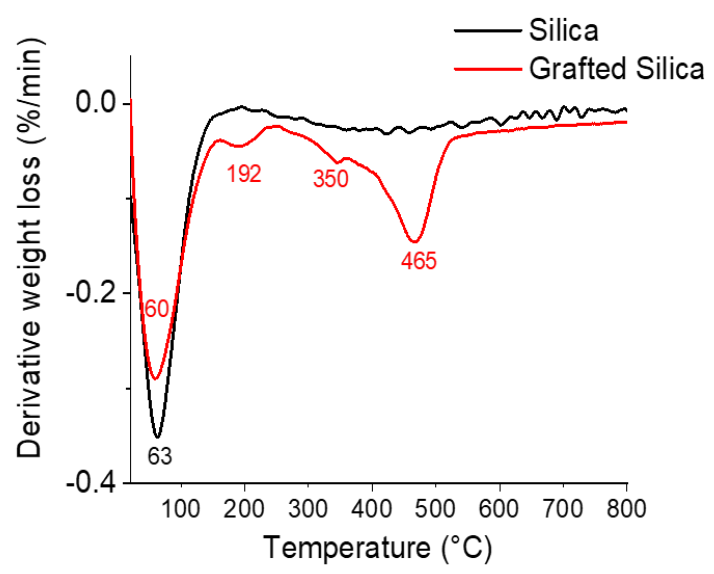

Fig. 1. Thermogravimetric analysis of the pure silica before grafting with SPI-1 (black) and of the grafted silica with SPI-1 (red). (A) corresponds to the weight loss (\%) and (B) corresponds to the derivative weight loss (\%/min).

The grafting mechanism between the silane groups and the surface of the silica is generally explained by considering two types of surface interactions [27]: i) physisorption of the silane on the silica via hydrogen bond between the alkoxy function and isolated and terminal silanols, ii) chemisorption via the formation of a covalent bond by condensation of alkoxy function and silanol group forming a Si-O-Si bond and releasing alcohol molecules. The FTIR spectrum of SPI-1/silica recorded at a temperature up to $200^{\circ} \mathrm{C}$ under secondary vacuum (Fig. 3(A)) shows that the decrease in the intensities of the characteristic vibration bands at $1390 \mathrm{~cm}^{-1}$ is weak ( 12\%) (Fig. 3(B)), confirming the low amount of physisorbed species and the high thermal stability of the remaining species. According to the literature [28], the high thermal stability of SPI-1 is typical of the covalent attachment of the silanes to the silica surface via the condensation of the alkoxy group with the silanol. Additionally, the comparison of the spectra of silica and SPI-1/silica recorded after evacuation at $200{ }^{\circ} \mathrm{C}$ reveal an irreversible decrease in the intensity of the $v(\mathrm{OH})$ band of the isolated silanol functions at $3744 \mathrm{~cm}^{-1}$ (of around $50 \%$ ) in the case of SPI-1/silica, showing the grafting of SPI-1 via the condensation of the alkoxy group with the isolated silanol. 
(A)

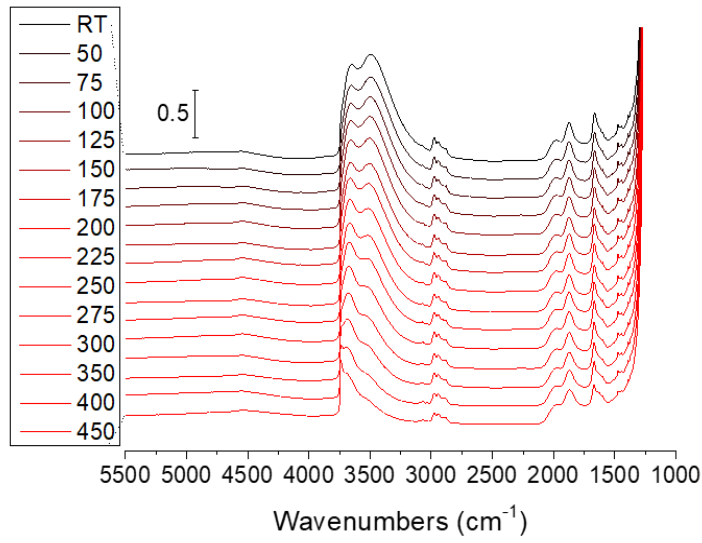

(B)

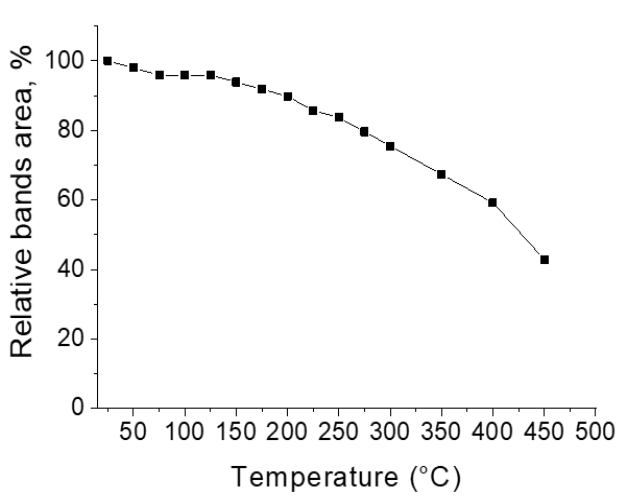

Fig. 3. (A) Evolution of the FTIR spectra of the grafted silica with SPI-1 as a function of temperature under vacuum $\left(P=10^{-6}\right)$ and $(B)$ evolution of the intensity of the vibration band at $1390 \mathrm{~cm}^{-1}$ attributed to the $\mathrm{C}-\mathrm{H}$ bending of the SPI-1 structure.

After confirming the covalent interactions and the thermal stability of SPI-1 on the surface of the silica particles, a free radical photopolymerization is conducted, whereby radical initiators are created on the surface upon UV irradiation and therefore triggering the polymerization. A kinetic study is performed first to monitor the rate of photopolymerization and measure the conversion of the monomer using in-situ FTIR. For this, few drops of trimethylolpropane triacrylate (TMPTA) are added on a SPI-1/Silica self-supported pellet (diameter of the pellet $=2 \mathrm{~cm}^{2}$ and thickness $=65 \mu \mathrm{m}$ ) without adding any external photoinitiator. The sample is placed in the in-situ FTIR cell, equipped with $\mathrm{KBr}$ windows and then subjected to polychromatic UV light and IR beam under controlled atmosphere (Ar), simultaneously. The evolution of the FTIR spectra along with the conversion yield are represented in Fig. 4. The conversion is measured by the evolution of the characteristic stretching band of the $\mathrm{C}=\mathrm{C}$ (at $1635 \mathrm{~cm}^{-1}$ ) of the monomer, and is calculated according to the following equation:

$$
\text { Conversion }(\%)=\left(A_{0}-A_{t}\right) / A_{0} * 100
$$

where $A_{0}$ and $A_{t}$ correspond to the band area at $t=0$ and $t=t$ of the photopolymerization, respectively. 
The results reveal a high yield conversion $\sim 75 \%$ after $300 \mathrm{~s}$ of irradiation confirming that the SPI-1 is still highly photoactive after its grafting on the surface of the silica. No polymerization observed when using pure silica without external photoinitiator under similar conditions. This result demonstrates the

(A)

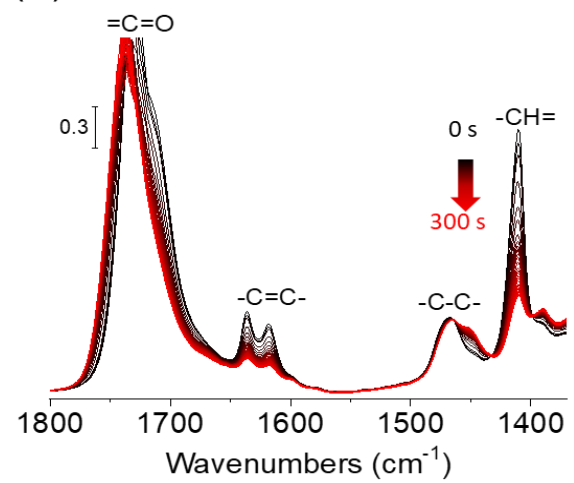

(B)

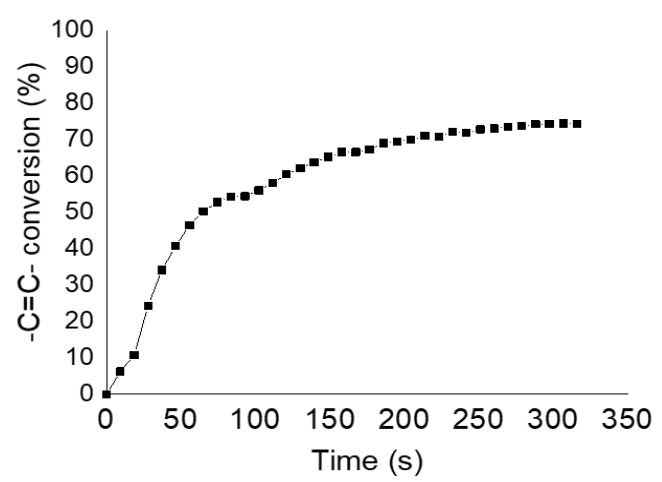

effectiveness of the grafted SPI-1.

Upon the successful synthesis of these hybrid materials via photopolymerization, their mechanical properties are examined using atomic force microscopy (AFM) and dynamic mechanical analysis (DMA). The topographic maps represented in Fig. 5 reveal smooth surfaces with nanometric roughness for the three films (Fig. 5(A)). The surface elastic modulus is calculated using DMT model in contact mechanic [29] and is found to be two to three times higher in SPI-1/silica@polymer film (2.1 $\pm 0.2 \mathrm{GPa}$ ) than that in silica@polymer film and pure polymer (Fig. 5(B) and 5(C)). Nanodomains (50 $\mathrm{nm}$ ) with high elastic modulus are also observed on the SPI-1/silica@polymer film. No differences are detected in the surface elastic modulus $(1.3 \pm 0.1 \mathrm{GPa})$ between the films of the pure polymer and the

Fig. 2. (A) Evolution of the FTIR spectra of SPI-1/silica pellet with 10 wt\% of TMPTA during photopolymerization. (B) Kinetic behavior of the photopolymerization of the TMPTA.

silica@polymer with a heterogeneous dispersion of silica agglomerates in this latter. This rise is attributed to the homogenous and high dispersion of the grafted silica particles whereas ungrafted particles are heterogeneously dispersed or agglomerated in the bulk of the polymer film. 

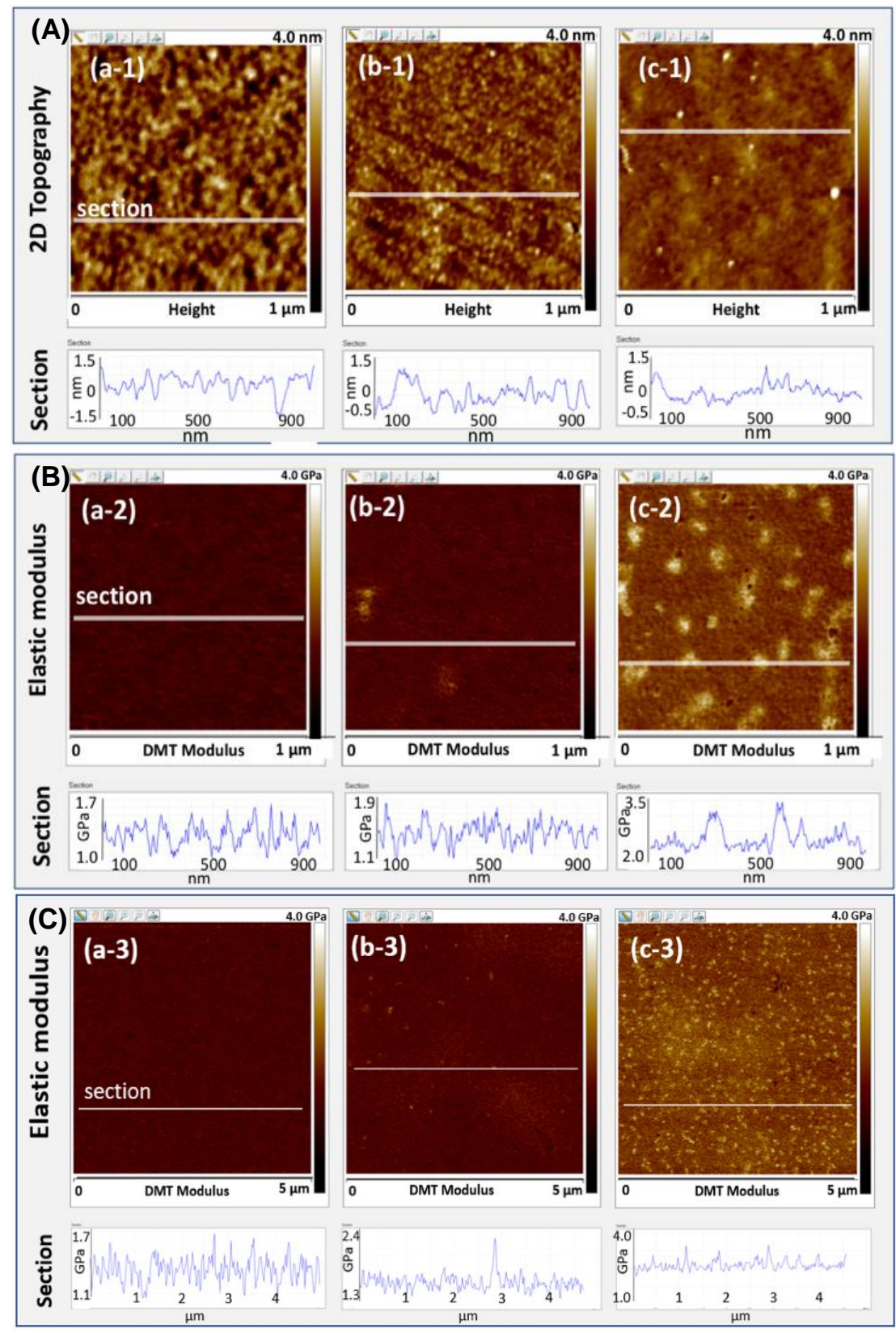

Fig. 3. (A) AFM topographic images at $1 \mu \mathrm{m}$ scale (z scale $4 \mathrm{~nm}$ ) and $(B-C)$ mechanical maps at two different scales $1 \mu \mathrm{m}$ and $5 \mu \mathrm{m}$ respectively ( $\mathrm{z}$ scale $4 \mathrm{GPa}$ ) of (a) pure polymer, (b) silica@polymer and (c) SPI-1/silica@polymer films. The white lines on the images correspond to the analysis range of the section's measurements illustrated at the bottom of each image.

The notable increase of the elastic modulus of the SPI-1/silica@polymer film is further investigated by the dynamic mechanical analysis since AFM is limited to report the properties of the surface. In fact, the DMA analysis scrutinizes the overall properties of the material. The elastic modulus ( $\left.E^{\prime}\right)$ of the pure polymer calculated from AFM and DMA are similar (about 1.3 GPa). Nevertheless, after adding ungrafted silica particles to the polymer film, a slight increase of the modulus ( $E^{\prime}$ ) is observed. An important increase of $E^{\prime}$ is calculated from the dynamic mechanical analysis for the SPI1/silica@polymer film. This surge, obtained also from AFM experiments, indicates that the enhancement of the mechanical properties is not only detected on the surface but in the vicinity of the 
polymer matrix. The evolution of the elastic modulus as a function of temperature is also exploited for the three films (Fig. 6). Its decrease is observed with the increase of the temperature for all the samples. This is due to the polymer softening upon heating. In accordance with AFM and DMA experiments, SPI-1/silica@polymer film has the highest modulus at different temperatures relative to pure polymer and ungrafted silica/polymer films. The pure polymer and the SPI-1/silica@polymer films behave similarly with the increase of temperature: a decrease of the storage modulus for both films is registered with the increase of the temperature. However, two remarkable decreases of the storage modulus of the silica@polymer film at $95^{\circ} \mathrm{C}$ and $110^{\circ} \mathrm{C}$ are distinguished. These shifts are probably due to the cracks of the film under the oscillation force indicating the presence of week interfacial regions between the polymer matrix and the filler particle which promotes the propagation of cracks. The absence of these cracks in the SPI-1/silica@polymer film is consistent with the enhancement of the interfacial interactions between silica nanoparticles and the surrounding polymer matrix. The highest elastic modulus is obtained when the content of SPI-1/Silica reaches $7.5 \%$ (Fig. S3).

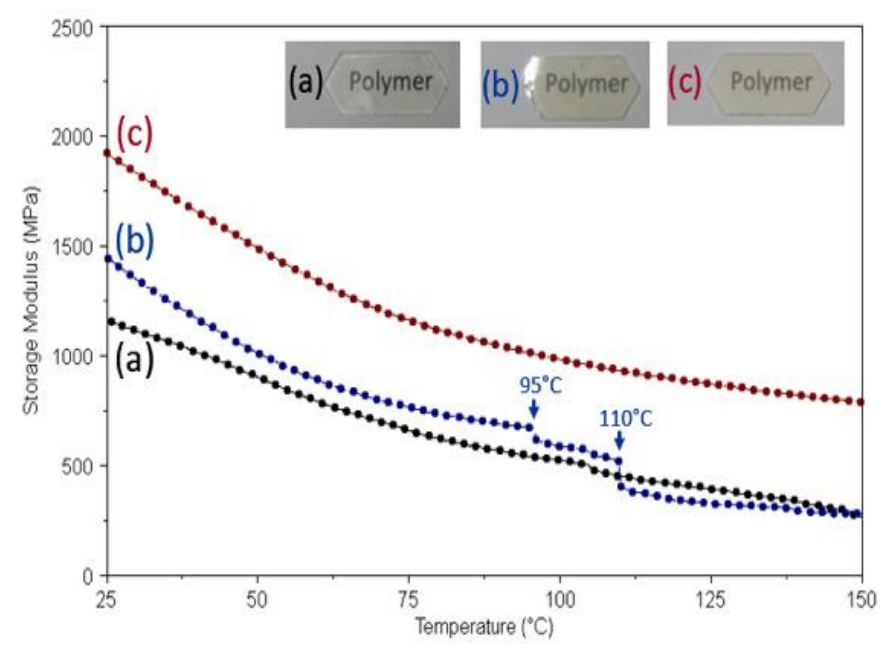

Fig. 4. Evolution of the storage modulus studied by DMA of (a) the pure polymer film, (b) ungrafted silica/polymer and (c) SPI-1/silica@polymer film. Silica content = $10 \mathrm{wt} . \%$. Sinusoidal strain of $10 \mu \mathrm{m}$ max at a frequency of $1 \mathrm{~Hz}$, heating rate 3 
To elucidate the origin of the prominent mechanical properties reported above, photopolymerization is studied in solution where the monomer is diluted in a solvent, to capture isolated particles. SPI-1/silica powder is dispersed in a solution of acetonitrile and then the photopolymerization is carried out under controlled atmosphere ( $\mathrm{Ar}$ ) and vigorous stirring in the presence of the monomer (TMPTA). The SPI-1/silica: monomer ratio is estimated by thermogravimetric analyses (TGA) and determined to be highest at 1:5 (Fig. S4). Accordingly, a ratio of $1: 5 \mathrm{wt} / \mathrm{wt}$. of silica/monomer is adapted. The collected powder is subjected to TEM-EDX analysis to examine its morphology. The TEM micrographs in Fig. 7(A-B) reveal a total coverage of silica particles by a shell layer of polymer. A close-up image on a single particle of $\mathrm{SiO}_{2}$. Fig. $7(\mathrm{C})$ shows the presence of a core-shell composite where silica particle is totally covered by an external shell layer of polymer. Furthermore, the EDX elemental maps in Fig. 7(D) represent the distribution of silicon, oxygen and carbon through which a consistent distribution of $\mathrm{Si}$ and $\mathrm{O}$ is observed where silica nanoparticles are present. A remarkable dispersion of carbon is noticed on the surface of silica nanoparticles, confirming thus the presence of a polymer shell layer on the surface of the silica nanoparticles.

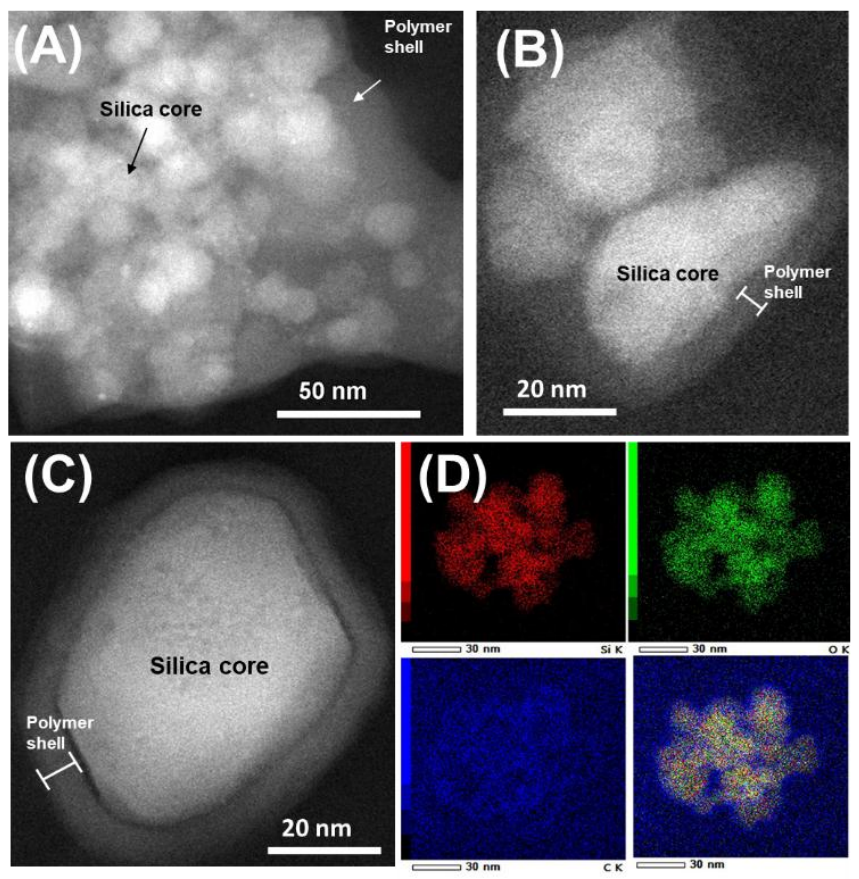

Fig. 5.(A-B-C) HAADF-STEM images and (D) EDX elemental mapping of SPI-1/Silica after photopolymerization of TMPTA in the presence of AcN. Si (red), O (green) and C (blue).

The core-shell like structure at the microscale level explains the mechanical behavior at the macroscale level of the polymer film containing SPI-1/Silica filler. The high dispersion of the SPI-1 on the silica surface as well as the covalent bond created between the silica surface and the polymer generate a highly stable hybrid network of ultra-dispersed silica particles with the polymer matrix. 


\section{Conclusion}

In conclusion, the efficiency of a newly synthesized silane-based photoinitiator (SPI-1) as a coupling agent for preparing hybrid SPI-1/silica@polymer materials via photopolymerization of trimethylolpropane triacrylate has been tested. Interestingly, the analysis of the mechanical properties of the hybrid materials shows a significant increase of the surface elastic modulus compared to that of the silica/polymer materials prepared using ungrafted silica as reference. The examination of the morphology of the SPI-1/silica@polymer single particles by HAADF-STEM indicates their core-shell structure resulting from a homogeneous polymerization at the silica surface. This behavior explains the increase of the elastic modulus by creating strong covalent bonds between the polymer and the silica surface through the grafting of the SPI-1 on this latter. The new procedure can be applied for preparing different core-shell materials for various applications, such as membranes, chromatographic colons, etc.

\section{Acknowledgment}

Authors acknowledge the Normandy region and European union for the financial support (Carnot project 3DZeoPol).

\section{Supplementary materials}

${ }^{1} \mathrm{H}$ NMR and FTIR data of the products synthetized in this work. Evolution of the elastic modulus versus the SPI-1/silica contents. Evolution of the polymer content versus SPI-1/silica@TMPTA ratio used for the photopolymerization of TMPTA in presence of SPI-1/silica suspension in acetonitrile.

\section{References:}

[1] Corrales, T., Catalina, F., Peinado, C., \& Allen, N. (2003). Free radical macrophotoinitiators: an overview on recent advances. Journal of Photochemistry and Photobiology A: Chemistry, 159(2), 103-114.

[2] Bag, D. S., \& Rao, K. B. (2010). Synthesis of UV-curable difunctional silane monomer based on 3-methacryloxy propyl trimethoxysilane (3-MPTS) and its UV-curing characteristics and thermal stability. Journal of applied polymer science, 115(4), 2352-2358.

[3] Zhou, S., Wu, L., You, B., \& Gu, G. (2009). Preparation, structure and properties of organicinorganic nanocomposite coatings. In. ACS Publications.

[4] Cooperstein, I., Layani, M., \& Magdassi, S. (2015). 3D printing of porous structures by UV-curable $\mathrm{O} / \mathrm{W}$ emulsion for fabrication of conductive objects. Journal of Materials Chemistry C, 3(9), 2040-2044.

[5] Vaezi, M., Seitz, H., \& Yang, S. (2013). A review on 3D micro-additive manufacturing technologies. The International Journal of Advanced Manufacturing Technology, 67(5-8), 1721-1754.

[6] Sangermano, M., Chiolerio, A., Marti, G., \& Martino, P. (2013). UV-Cured Acrylic Conductive Inks for Microelectronic Devices. Macromolecular materials and engineering, 298(6), 607-611.

[7] Chang, C.-C., Oyang, T.-Y., Hwang, F.-H., Chen, C.-C., \& Cheng, L.-P. (2012). Preparation of polymer/silica hybrid hard coatings with enhanced hydrophobicity on plastic substrates. Journal of non-crystalline solids, 358(1), 72-76.

[8] Zhang, L., Ren, Y., Peng, S., Guo, D., Wen, S., Luo, J., \& Xie, G. (2019). Core-shell nanospheres to achieve ultralow friction polymer nanocomposites with superior mechanical properties. Nanoscale, 11(17), 8237-8246. 
[9] Guo, D., Li, J., Xie, G., Wang, Y., \& Luo, J. (2014). Elastic properties of polystyrene nanospheres evaluated with atomic force microscopy: size effect and error analysis. Langmuir, 30(24), 7206-7212.

[10] Zhou, J., Allonas, X., Ibrahim, A., \& Liu, X. (2019). Progress in the development of polymeric and multifunctional photoinitiators. Progress in Polymer Science, 99, 101165.

[11] El-Roz, M., Lalevee, J., \& Fouassier, J. (2011). A search for new radical sources in photoinitiating systems. Current Trends in Polymer Science, 15, 1-13.

[12] El-Roz, M., Lalevée, J., Morlet-Savary, F., Allonas, X., \& Fouassier, J. P. (2008). Radical and cationic photopolymerization: New pyrylium and thiopyrylium salt-based photoinitiating systems. Journal of Polymer Science Part A: Polymer Chemistry, 46(22), 7369-7375.

[13] Li, X., Liu, Z., Hong, P., Chen, L., \& Liu, X. (2020). Synthesis of organic and inorganic hybrid nanoparticles as multifunctional photoinitiator and its application in UV-curable epoxy acrylate-based coating systems. Progress in Organic Coatings, 141, 105565.

[14] Sahin, M., Krawczyk, K. K., Roszkowski, P., Wang, J., Kaynak, B., Kern, W., Schlögl, S., \& Grützmacher, H. (2018). Photoactive silica nanoparticles: Influence of surface functionalization on migration and kinetics of radical-induced photopolymerization reactions. European Polymer Journal, 98, 430-438.

[15] Schwalm, R. (2006). UV coatings: basics, recent developments and new applications. Elsevier.

[16] El-Roz, M., Lalevée, J., Allonas, X., \& Fouassier, J. P. (2009). Mechanistic investigation of the silane, germane, and stannane behavior when incorporated in type I and type II photoinitiators of polymerization in aerated media. Macromolecules, 42(22), 8725-8732.

[17] Deng, J., Wang, L., Liu, L., \& Yang, W. (2009). Developments and new applications of UVinduced surface graft polymerizations. Progress in Polymer Science, 34(2), 156-193.

[18] Zhao, B., \& Brittain, W. J. (2000). Synthesis of polystyrene brushes on silicate substrates via carbocationic polymerization from self-assembled monolayers. Macromolecules, 33(2), 342348.

[19] Dworjanyn, P. A., Garnett, J. L., Khan, M. A., Maojun, X., Meng-Ping, Q., \& Nho, Y. C. (1993). Novel additives for accelerating radiation grafting and curing reactions. Radiation Physics and Chemistry, 42(1-3), 31-40.

[20] Chen, R., Zhu, S., \& Maclaughlin, S. (2008). Grafting acrylic polymers from flat nickel and copper surfaces by surface-initiated atom transfer radical polymerization. Langmuir, 24(13), 68896896.

[21] Xu, G. C., Li, A. Y., Zhang, L. D., Wu, G. S., Yuan, X. Y., \& Xie, T. (2003). Synthesis and characterization of silica nanocomposite in situ photopolymerization. Journal of applied polymer science, 90(3), 837-840.

[22] Bauer, F., Flyunt, R., Czihal, K., Langguth, H., Mehnert, R., Schubert, R., \& Buchmeiser, M. R. (2007). UV curing and matting of acrylate coatings reinforced by nano-silica and microcorundum particles. Progress in Organic Coatings, 60(2), 121-126.

[23] Wu, J., Ling, L., Ma, G., \& Wang, B. (2014). A comparative study of grafting steps on the preparation and properties of modified nanosilica for UV-curable coatings. Journal of Coatings Technology and Research, 11(5), 717-725.

[24] Ranjbar, Z., \& Rastegar, S. (2009). The influence of surface chemistry of nano-silica on microstructure, optical and mechanical properties of the nano-silica containing clear-coats. Progress in Organic Coatings, 65(1), 125-130.

[25] EL ROZ, M. L., Sami ; TELEGEIEV, Igor (2017). New photoinitiator based on bifunctional silane. CNRS innovation. https://www.cnrsinnovation.com/catalogue-cnrs/new-photoinitiator-basedon-bifunctional-silane/

[26] El Roz, M., Lakhdar, S., \& Telegeiev, I. (2021). U.S. Patent Application No. 16/764,805.

[27] El-Roz, M., Thibault-Starzyk, F., Wehmeier, A., Maschke, D., \& Blume, A. (2016). Silica-silane reaction: Deciphering the silica-silane reaction mechanism for the development of a new generation of low rolling reistsnace tires. Tire technology international, 2016(43), 93-94.

[28] El-Roz, M., Thibault-Starzyk, F., \& Blume, A. (2014). Infrared study of the silica/silane reaction. Part II. KGK Kautschuk, Gummi, Kunststoffe, 67(5), 53-57.

[29] Derjaguin, B. V., Muller, V. M., \& Toporov, Y. P. (1975). Effect of contact deformations on the adhesion of particles. Journal of Colloid and Interface Science, 53(2), 314-326. 